\title{
LACTANCIA MATERNA: DECISION INTELIGENTE, MULTIPLES BENEFICIOS Y FUTURO PROTEGIDO
}

\author{
Carmen Dávila Aliaga ${ }^{1}$
}

\begin{abstract}
RESUMEN
El mejor alimento para el recién nacido es la leche materna, dada la evidencia de ventajas médicas y del neurodesarrollo a corto y largo plazo, Debe ser considerada una estrategia de salud pública para reducción de morbimortalidad neonatal, mejora la calidad de vida a corto y largo plazo. La leche materna, también conocida como la sangre blanca, compuesta por aproximadamente 258 componentes, desde células vivas, macro y micronutrientes, hormonas, moléculas del sistema de defensa, inmunoglobulinas, bacterias, etc; posee gran variabilidad en relación al momento dentro de la mamada, hora del día, edad del bebé, de acuerdo a las necesidades del bebé entre otros. Las ventajas se extienden a la madre y son múltiples. Para aquellos casos en los que es imposible contar con leche materna debemos poder ofrecer la opción de recibir leche pasteurizada de un banco de leche. Actualmente el ministerio de salud y las instituciones de salud venimos trabajando para acreditar como hospital amigo de la madre y del niño, velando por el cumplimiento de los 10 pasos para una lactancia materna exitosa, fomentando y apoyando la lactancia materna.
\end{abstract}

Palabras clave: Amamantamiento, leche humana, lactancia (Fuente: DeCS BIREME).

\section{BREASTFEEDING: INTELLIGENT DECISION, MULTIPLE BENEFITS AND PROTECTED FUTURE}

\begin{abstract}
The best food for the newborn is breast milk, given the evidence of short and long-term medical and neurodevelopmental benefits. It should be considered a public health strategy to reduce neonatal morbidity and mortality, improve quality of life in short and long term. Breast milk, also known as white blood, consists of approximately 258 components, from living cells, macro and micronutrients, hormones, defense system molecules, immunoglobulins, bacterias, etc; has great variability in relation to the moment within the breastfeeding, time of day, age of the baby, according to the needs of the baby among others. The advantages extend to the mother and are multiple. For those cases where it is impossible to get breast milk, we must be able to offer the option of receiving pasteurized milk from a milk bank. Currently the Ministry of Health and health institutions have been working to accredit as a hospital friend of the mother and child, ensuring compliance with the 10 steps for successful breastfeeding, encouraging and supporting breastfeeding.
\end{abstract}

Key words: Breastfeeding; Human milk; Lactation (Source: MeSH NLM).

\section{INTRODUCCIÓN}

La tasa de mortalidad neonatal (TMN) mundial se redujo en $50 \%$ en los últimos 25 años. Las causas más frecuentes son sepsis, meningitis, neumonía, diarrea, prematuridad y malformaciones congénitas ${ }^{1,2}$.

La ampliación de la lactancia materna a nivel universal podría evitar 823,000 muertes anuales de niños menores de 5 años y 20,000 muertes anuales por cáncer de mama ${ }^{3}$. En nuestro país, la TMN se redujo, entre 1990 y 2015, en un $37 \%$ sin embargo el componente duro de la mortalidad infantil es la neonatal, que llega a ser el $56 \%$ de la mortalidad infantil. ${ }^{1}$ Dentro de las políticas y estrategias de mayor impacto recomendadas para disminuir esta problemática, esta apoyar, promocionar y proteger la lactancia materna, que contribuye a reducir la morbilidad y mortalidad neonatal ${ }^{4}$.

La leche materna brinda la alimentación ideal para los lactantes, garantiza su crecimiento y desarrollo, además tiene múltiples beneficios para la madre, la información científica es en la actualidad contundente, disminuye la morbilidad y la mortalidad infantil, se asocia a menor número y gravedad de infecciones, mejor coeficiente de inteligencia, menor incidencia de alergias, efecto protector contra sobrepeso y diabetes; prevención del cáncer en los bebés y sus madres, entre muchos otros. Adicionalmente se reportan indudables beneficios económicos, menor número de hospitalizaciones, alimento siempre disponible, no se contamina en procesos de preparación, etc ${ }^{5}$.

\section{ASPECTOS RELEVANTES EN LA COMPOSICION DE LA LECHE MATERNA}

La leche humana es un sistema complejo, contiene múltiples nutrientes y gran variabilidad ${ }^{6}$. La concentración de algunos nutrientes varía en función al momento de la lactancia, duración de la misma, hora del día y necesidades del bebé; mientras que otros nutrientes son relativamente estables. La energía contenida en la leche humana es $20 \mathrm{Kcal} / \mathrm{oz}(0,67 \mathrm{Kcal} / \mathrm{ml})$. El contenido proteíco es muy alto al inicio en los recién nacidos a término $2,3 \mathrm{~g} / \mathrm{dl}$ (calostro). Estos niveles disminuyen en las siguientes dos a cuatro semanas hasta $1,8 \mathrm{~g} / \mathrm{dl}$. Las proteínas proporcionan el $8 \%$ de las necesidades calóricas y aseguran un estado

Médico Pediatra Neonatóloga, docente de la Universidad Federico Villarreal. Directora Ejecutiva de Investigación, Docencia y Atención en Neonatología del Instituto Nacional Materno Perinatal. Lima-Perú 
proteico adecuado del lactante amamantado durante el primer año ${ }^{7}$.

Aproximadamente $70 \%$ de las proteínas en la leche humana están en la fracción de suero soluble y $30 \%$ en la fracción de caseína insoluble. La alta proporción de proteína de suero en la leche humana es beneficiosa para los bebés porque el suero se digiere más fácilmente y se asocia con un vaciado gástrico más rápido ${ }^{8}$. La principal proteína de suero de leche humana es la lactoalbúmina. Además, tiene niveles altos de cistina (necesaria para sintetizar el antioxidante glutatión) y taurina (necesaria para la conjugación de la bilis y el desarrollo del cerebro 9. La lactoferrina, la lisozima y la inmunoglobulina $A$ secretora son proteínas de suero humano específicas que mejoran la defensa del huésped ${ }^{9,10}$.

En la leche humana, aproximadamente el $20 \%$ del nitrógeno total está en forma de compuestos no proteicos, como nucleótidos que son importantes para las funciones gastrointestinal, inmune y metabólica; aminoácidos libres y urea ${ }^{11}$.

Las grasas representan aproximadamente el $50 \%$ de las calorías en la leche humana. Los contenidos lipídicos y energéticos de la leche humana aumentan durante la lactancia. Esto fue demostrado en un estudio que comparó muestras de leche humana de madres que habían amamantado durante más de 1 año con las de madres que habían amamantado durante dos a seis meses ${ }^{12}$.

La leche humana facilita la digestión y absorción de lípidos por su organización de lípidos en triglicéridos de glóbulos grasos de leche ${ }^{13}$. Es única en su contenido de ácidos grasos de cadena muy larga tales como ácido araquidónico y ácido docosahexaenoico, los cuales se han asociado con una mejor cognición, crecimiento y visión; estos son derivados de los ácidos grasos esenciales, ácidos linoleico y linolénico ${ }^{14,15}$.

El contenido lipídico total de la leche humana no se ve afectado por la dieta materna, aunque puede estar correlacionado directamente con las reservas maternas de grasa corporal. A pesar de la variabilidad entre las mujeres, el contenido de lípidos de la leche humana es adecuado para las necesidades nutricionales del lactante durante la lactancia ${ }^{5}$.

Los carbohidratos de la leche humana se componen principalmente de lactosa, con una pequeña proporción constituida por oligosacáridos, estos son importantes en la defensa del huésped, ya que sus estructuras imitan a los receptores de antígenos bacterianos específicos ${ }^{16}$.

Las concentraciones de calcio y fósforo en la leche humana son relativamente constantes durante la lactancia, de gran biodisponibilidad. Los minerales están ligados a proteínas digeribles y también están presentes en estados complejos e ionizados. En un estudio, el seguimiento a los ocho años de edad mostró que los niños amamantados en comparación con los que no habían amamantado tenían una masa ósea significativamente mayor ${ }^{5,16}$.

La baja carga de solutos, lipasa, factores tróficos y de maduración sobre el sistema gastrointestinal, la mejor absorción de las grasas, oligosacáridos que participan en el sistema de defensa y otros factores previenen de enterocolitis necrosante.

La leche materna contiene elementos esenciales para el desarrollo neurológico de los lactantes ${ }^{16}$.

El tipo de nacimiento inicialmente establece si la flora intestinal de la madre (parto vaginal) o la flora de la piel de los asistentes de parto (cesárea) dominarán los colonizadores iniciales ${ }^{17,18}$ los cuales inducen una respuesta inmune importante en el lactante.

El tipo de alimentación es el segundo determinante del microbioma infantil. Los infantes amamantados mantienen diferencias microbianas persistentes, debido a los efectos de los oligosacáridos de la leche humana $(\mathrm{OLH})^{18,19}$.

La leche humana contiene una variedad de azúcares; un $8 \%$ de su valor calórico es en forma de OLH no digeribles, que funcionan como prebióticos para apoyar el crecimiento de bacterias específicas, Bifidobacterium infantis longum biovar, que ha evolucionado para expresar las enzimas necesarias para la utilización de los $\mathrm{OLH}^{17,20}$.

Hay especificidad en la interacción entre la leche materna y el microbioma infantil, lo cual induce efectos de bacterias sobre el metabolismo y la inmunidad del infante.

El eje enteromamario de la madre permite que las células dendríticas intestinales de la madre tomen las bacterias individuales del lumen y las transporten a los folículos linfoides ${ }^{21}$ del intestino, los linfocitos y las células dendríticas programadas vuelven a entrar en la circulación antes de volver al intestino a través de la interacción entre sus integrinas $\beta 7$ y la expresión local de la molécula de adhesión celular de la mucosa vascular (MAdCAM-1). La MAdCAM-1 se expresa en el endotelio mamario durante el embarazo, lo que permite la absorción selectiva de células programadas en el intestino. El efecto del tráfico enteromamario incluyen la liberación de células dendríticas que contienen bacterias intestinales maternas vivas $^{17}$

La leche materna contiene, células inmunes del fenotipo relacionado con el intestino (células $\gamma \delta$, células $\beta 7+$ ) que han madurado en el intestino. Las citoquinas de la leche materna también varían en función de las experiencias inmunológicas de la madre.

La cesárea, los antibióticos perinatales y el no amamantar son los tres factores que afectan este proceso de impresión que evoluciona conjuntamente. Además 
de los cambios mediados a través de la flora, los componentes individuales de la leche materna podrían afectar directamente la programación epigenética del lactante. El efecto adverso de los polimorfismos de los receptores $y$ activados por proliferadores de peroxisomas en la adiposidad y el metabolismo se evita mediante la lactancia materna ${ }^{17,22}$.

La lactoferrina, un importante componente de la leche materna, une receptores CpG bacterianos y evoca las respuestas a la flora NF-kB de la mucosa. Microvesículas llamadas exosomas se segregan en la leche materna $y$ podrían inhibir la sensibilización atópica que depende de la experiencia inmunitaria materna ${ }^{23}$.

Los glóbulos de grasa de la leche materna contienen muchos micro-ARN segregados, cuya expresión está modulada por la dieta materna. Existe también evidencia de que en la leche materna se segregan células madre multipotenciales y pueden persistir dentro de los lactantes.

La microbioma (número total de microorganismos y su material genético) tiene la capacidad de regular las respuestas de hospedero en la infancia, depende de especies bacterianas individuales, que modulan la polarización de las células $T$ y la regulación inmune, las respuestas metabólicas, la adipogénesis e incluso el desarrollo del cerebro y el funcionamiento cognitivo ${ }^{21,24}$.

La colonización anormal tiene un efecto dañino a largo plazo sobre la homeostasis inmune y metabólica. Por tanto, la leche de la madre transmite su propio microbioma y respuestas inmunes y también proporciona prebióticos específicos para apoyar el crecimiento de las bacterias benéficas en el bebé.
La leche materna está compuesta de células y sustancias celulares y no celulares que brindan protección pasiva y activa contra virus, bacterias, enterotoxinas, hongos y protozoarios ${ }^{17,25}$

\section{VENTAJAS DE LA LECHE MATERNA EN EL BEBÉ}

La leche materna es el alimento ideal para el recién nacido y genera muchos beneficios para toda su vida, ${ }^{26}$ como: (Tabla 1) Mejora el vaciamiento gástrico, favorece una rápida expulsión de meconio, menos reflujo gastroesofágico. Estimulación activa del sistema inmunitario digestivo y sistémico del lactante. Mejor respuesta inmune a las vacunas.

Se le asocia a reducción del riesgo de infecciones (gastroentéricas, respiratorias, urinarias, generalizadas) y también el de muerte súbita del lactante ${ }^{26-28}$.

Los lactantes que recibieron lactancia materna exclusiva los primeros 6 meses, tinen menos enfermedades, como: enterocolitis necrotizante, enfermedad celíaca, colitis ulcerosa, Crohn, diabetes tipo I, artritis reumatoide, esclerosis múltiple, algunos tipos de cáncer como leucemias (leucemia linfocítica aguda y leucemia mieloide aguda), cáncer de mama premenopáusico o posmenopáusico en la edad adulta y enfermedades alérgicas (dermatitis atópica, rinitis y asma) ${ }^{29-43}$.

En niños con retardo de crecimiento intrauterino, que presentan alteraciones de la función digestiva secundaria a hipoxia, con alteración de producción enzimática y por ende reducción de capacidad de absorción de nutrientes, la lactancia materna logra vencer estas barreras con éxito demostrado.

Tabla 1. Beneficios de lactancia materna en el bebé.

\begin{tabular}{|c|c|c|c|c|c|c|}
\hline EFECTO ESTUDIADO & $\begin{array}{l}\text { REDUCCION } \\
\text { DE RIESGO }\end{array}$ & $\begin{array}{c}N^{\circ} \mathrm{DE} \\
\text { ESTUDIOS }\end{array}$ & RR/OR & IC 95\% & Observaciones & Bibliografía \\
\hline Diarrea & $50 \%$ & 23 & RR 0.37 & $0.27-0.5$ & Lactancia mayor 6 meses & 28 \\
\hline Infecciones resp baja & $72 \%$ & 1 & 0.28 & $0.14-0.5$ & $\mathrm{LME}>4 \mathrm{~m}$ & 28 \\
\hline Infecciones resp & $33 \%$ & 16 & RR 0.68 & $0.6-0.7$ & Lactancia mayor 6 meses & 29 \\
\hline $\begin{array}{l}\text { Mortalidad debida a } \\
\text { enfermedades infecciosas }\end{array}$ & SI & 3 & OR 0.22 & $0.14-0.34$ & Lactancia exclusiva vs parcial & 27 \\
\hline Rinitis alérgica & SI & 29 & OR 0.91 & $0.85-0.98$ & & 43 \\
\hline Asma & $9 \%$ & 29 & & $0.02-0.15$ & & 43 \\
\hline Asma & $40 \%$ & 1 & 0.6 & $0.43-0.82$ & LME $>3 m$ en historia de atopía & 29 \\
\hline Otitis media recurrente & $77 \%$ & 1 & 1.95 & $1.06-3.59$ & $\mathrm{LME}>6 \mathrm{~m}$ vs $<6 \mathrm{~m}$ & 31 \\
\hline Otitis media & $50 \%$ & 1 & 0.5 & 0.36 .07 & $\mathrm{LME} \geq 6 \mathrm{~m}$ & 29 \\
\hline Otitis media & $\mathrm{SI}$ & 11 & OR 0.67 & $0.62-0.72$ & & 41 \\
\hline Malaoclusión dental & $\mathrm{SI}$ & 41 & OR 0.32 & $0.25-0.4$ & LME vs parcial & 61 \\
\hline Diabetes tipo 1 & $30 \%$ & 1 & 0.71 & $0.54-0.93$ & LME $>3 \mathrm{~m}$ & 38 \\
\hline Diabetes tipo 2 & $40 \%$ & 1 & 0.61 & $0.44-0.85$ & LME >3m & 38 \\
\hline Leucemia (LLA) & $20 \%$ & 1 & 0.8 & $0.71-0.91$ & LME $>6 m$ & 39 \\
\hline Leucemia (LMA) & $15 \%$ & 1 & 0.85 & $0.73-0.98$ & LME $>6 m$ & 40 \\
\hline Obesidad & $24 \%$ & 1 & 0.76 & $0.67-0.86$ & & 29 \\
\hline Inteligencia & SI & 16 & & & $\begin{array}{l}\text { Mejora 3-7 puntos en score de } \\
\text { Inteligencia }\end{array}$ & 49 \\
\hline
\end{tabular}

LME: lactancia materna exclusiva 
Los prematuros que representan la población más expuesta a eventos adversos, mostró contundentes efectos positivos al alimentarse con leche humana: Menor tiempo en lograr una nutrición enteral completa y capacidad de absorción de nutrientes ${ }^{44,45}$ tasas más bajas de sepsis y enterocolitis necrosante ${ }^{46,47}$ menores tasas de retinopatía severa del prematuro, ${ }^{48}$ mejor valoración del neurodesarrollo a largo plazo, ${ }^{49}$ mejores puntajes en evaluaciones de comportamiento, capacidad motora y mental en seguimiento a 18 y 30 meses ${ }^{50,51}$.

Los resultados de evaluaciones de inteligencia son mejores en lactantes amamantados por 3 meses a más ${ }^{52-55}$. Mortenson y cols en su estudio muestra que los niños amamantados durante 6 a 9 meses tienen un coeficiente intelectual que supera en unos 6 puntos al de los niños amamantados durante menos de un mes ${ }^{56}$. Mejor agudeza visual en la etapa escolar, desarrollo intelectual y social ${ }^{57}$.

En seguimiento de prematuros hasta adolescentes se encontró tasas más bajas de alteración en el metabolismo de la insulina, mejores concentraciones de lipoproteínas de baja densidad, leptina, menos infarto de miocardio e hipertensión en la edad adulta ${ }^{58,59}$. Owen realiza una revisión sistemática en la que se concluye con la disminución de riesgo de obesidad asociado a lactancia materna ${ }^{60}$.

Estudios a largo plazo han demostrado menor prevalencia de caries, de anomalías dentofaciales, como malaoclusión dental ${ }^{61}$.

\section{VENTAJAS DE AMAMANTAR EN LA SALUD MATERNA}

El amamantamiento dentro de la primera hora de vida favorece la liberación de oxitocina, favorece el alumbramiento, disminuye el sangrado uterino, favorece la involución uterina y por ende previene anemia $^{62}$. La impronta que ocurre en ese momento no sólo favorecerá la colonización bacteriana, tal vez marco su neurodesarrollo.
Lactancia materna exclusiva durante los primeros 6 meses, inhibe la ovulación durante un largo período, amenorrea por lactancia, esto permite espaciar los embarazos. ${ }^{63}$

Permite la recuperación progresiva del peso antes de la gestación dado que la producción de leche genera gasto energético $0^{64,65}$.

En las madres sin antecedentes de diabetes gestacional, la lactancia materna se asoció con una disminución del riesgo de diabetes mellitus tipo 2. Un metanálisis de seis estudios de cohortes sobre la diabetes tipo 2 mostró una razón de momios (odds ratio) de 0.68 (IC 95\% $0.57-0.82$ ) también se reporta reducción de riesgo de enfermedades cardiovasculares, como hipertensión, el factor de protección se encuentra en relación al tiempo de lactancia ${ }^{69,70}$.

Se reportan cambios positivos en el metabolismo de las grasas y el calcio. Se ha demostrado que a largo plazo las mujeres que amamantan tienen menos riesgo de osteoporosis $\mathrm{y}$, por tanto, de fracturas vertebrales $\mathrm{y}$ de cadera en la posmenopausia. También disminuye la incidencia de depresión en el puerperio ${ }^{71,72}$.

Se reduce el riesgo de padecer cáncer mamario hasta en $4,3 \%$ por cada 12 meses de lactancia materna, a lo que se adiciona $7 \%$ por nacimiento. La protección contra el cáncer de mama para la madre lactante podría estar mediada a través de la modulación del receptor activado del proliferador de peroxisoma. Cada incremento de 12 meses en período de lactancia se asoció con una reducción del 4,3\% (IC 95\% 2.9-6.8) en la incidencia de cáncer de mama invasivo. Este análisis ajustó factores de confusión, como la paridad y se excluyeron a las mujeres nulíparas. Los resultados no varían sustancialmente de acuerdo con el estado menopáusico. Este metanálisis sugiere una magnitud más alta de protección hasta 7\%, cuando el análisis se limitó a 14 estudios con un ajuste por paridad y la exclusión de las mujeres nulíparas, y se comparó lactancia de larga duración (mayor de 12 meses) con duraciones más cortas (IC 95\% $3-11)^{73,74}$. El cáncer de ovarios y útero también es menos frecuente en madres que han dado de lactar.

Tabla 2. Beneficios en la madre que amamanta

\begin{tabular}{|c|c|c|c|c|c|c|}
\hline EFECTO ESTUDIADO & $\begin{array}{c}\text { REDUCCIÓN } \\
\text { DE RIESGO }\end{array}$ & $\begin{array}{c}\mathrm{N}^{\circ} \mathrm{DE} \\
\text { ESTUDIOS }\end{array}$ & RR/OR & IC $95 \%$ & OBSERVACIONES & BIBLIOGRAFÍA \\
\hline Diabetes tipo II & $\mathrm{Si}$ & 6 & 0.68 & $0.57-0.82$ & LM prolongada & 66 \\
\hline Cáncer de ovario & SI & 41 & 0.7 & $0.64-0.75$ & LM prolongada & 73 \\
\hline Cáncer de mama & $\mathrm{SI}$ & 76 & 0.81 & $0.77-0.86$ & LM prolongada & 73 \\
\hline Cáncer de mama & SI & & 0.72 & $0.65-0.8$ & $\mathrm{LM}>12 \mathrm{~m}$ & 74 \\
\hline Cáncer de ovario & $\mathrm{SI}$ & & 0.72 & $0.54-0.97$ & $\mathrm{LM}>12 \mathrm{~m}$ & 74 \\
\hline Amenorrea de lactancia & SI & 13 & 1.1.8 & $1.04-1.32$ & LM prolongada & 73 \\
\hline Obesidad/ Sobrepeso & SI & 113 & 0.74 & $0-7-0.78$ & Lactancia prolongada & 69 \\
\hline Diabetes tipo 2 & $\mathrm{SI}$ & 11 & 0.45 & $0.49-0.86$ & LME vs parcial & 69 \\
\hline Artritis reumatoidea & $\mathrm{SI}$ & & 0.8 & $0.8-0.1$ & $\mathrm{LM}>12 \mathrm{~m}$ & 75 \\
\hline Artritis reumatoidea & SI & & 0.5 & $0.3-0.8$ & $\mathrm{LM}>24 \mathrm{~m}$ & 75 \\
\hline Hipertensión arterial & SI & & 0.89 & $0.84-0.93$ & LM 12-23m & 67 \\
\hline Hiperlipidemia & $\mathrm{SI}$ & & 0.81 & $0.76-0.87$ & LM 12-23m & 68 \\
\hline Enf. Cardiovascular & SI & & 0.9 & $0.85-0.96$ & LM 12-23m & 68 \\
\hline
\end{tabular}

LME: lactancia materna exclusiva LM: lactancia materna 
Tabla 3. 10 pasos para una lactancia materna exitosa.

\begin{tabular}{|c|c|}
\hline Paso 1 & $\begin{array}{l}\text { Existencia de una Política escrita de Promoción y } \\
\text { Protección de la lactancia }\end{array}$ \\
\hline Paso 2 & Capacitación del personal de salud \\
\hline Paso 3 & Entrega de información a gestante \\
\hline Paso 4 & Realización del contacto piel a piel \\
\hline Paso 5 & $\begin{array}{l}\text { Mostrar a las madres como amamantar y como } \\
\text { mantener la lactancia aun si ellas deben separarse de } \\
\text { sus hijos }\end{array}$ \\
\hline Paso 6 & $\begin{array}{l}\text { No dar a los recién nacidos alimento o líquido que no } \\
\text { sean leche materna }\end{array}$ \\
\hline Paso 7 & $\begin{array}{l}\text { Alojamiento conjunto durante la estancia en el } \\
\text { establecimiento de salud }\end{array}$ \\
\hline Paso 8 & Lactancia materna a demanda \\
\hline Paso 9 & No dar chupones ni biberones a recién nacidos \\
\hline Paso 10 & $\begin{array}{l}\text { El establecimiento tiene contacto con Grupos de } \\
\text { Apoyo }\end{array}$ \\
\hline
\end{tabular}

Karlson reporta menor incidencia de artritis reumatoide en las mujeres que lactan a sus niños, proporcional al tiempo de lactancia ${ }^{75}$ (Tabla 2).

A todo esto se suma una estrecha y grata relación que se establece entre la madre y el niño, así como el placer de amamantar.

\section{VENTAJAS PARA LA FAMILIA Y LA SOCIEDAD}

La lactancia materna reduce los gastos en salud por disminución de la morbilidad y, por esta misma razón, disminuye el ausentismo laboral de los padres a los centros laborales. Preserva el medioambiente, reduce la producción de residuos y el consumo eléctrico e hídrico. Contribuye a la economía familiar, al eliminar las inversiones en fórmulas y accesorios para la lactancia artificial y en terapias.

Un análisis detallado de los costos basados en el informe del Centro de prácticas basado en evidencia de la agencia para investigación en cuidados de la salud y calidad (AHRQ) concluyó que si el $90 \%$ de las madres estadounidenses cumplen con la recomendación de amamantar exclusivamente por 6 meses, habría un ahorro de $\$ 13$ mil millones de dólares por año. En Estados Unidos podrían salvarse 900 niños/año si las madres dieran lactancia materna exclusiva durante 6 meses $^{74}$.

\section{LACTANCIA MATERNA: INICIATIVA MUNDIAL}

La Academia Americana de Pediatría recomienda lactancia materna exclusiva por 6 meses, seguida de ablactancia con lactancia como mínimo hasta 1 año. El $90 \%$ de las muertes infantiles en el mundo ocurren en 42 países en desarrollo, con lactancia materna exclusiva durante 6 meses y destete luego del año de vida pueden prevenirse un millón de muertes por año, lo que representaría reducción del $13 \%$ de la mortalidad infantil en el mundo.

Los recién nacidos que son colocados en el seno durante la primera hora de vida, tienen una menor probabilidad de morir en el período neonatal. En los estudios de Edmond y cols, así como en el de Mullany y cols. que incluyeron cerca de 34,000 recién nacidos, muestran que el riesgo de muerte se incrementa a medida que existe demora en el inicio de la $\mathrm{LM}^{77,78}$. La importancia del inicio temprano de la LM ha sido reconocida por la OMS, la que recomienda que todos los recién nacidos a término, con una edad gestacional superior a las 32 semanas o que nacen con un peso mayor a 1500 gramos, sean colocados al seno durante la primera hora de vida.

Si la leche materna no está disponible debería utilizarse leche de donante pasteurizada, la cual tiene todo un proceso de alta calidad, el cual consiste luego de su recepción un meticuloso control de cadena de frío, análisis sensorial, fisicoquímico, pasteurización propiamente dicha y control microbiológico de cada frasco de leche donada; luego de lo cual se procede a la distribución de acuerdo a la prescripción médica según las necesidades del paciente ${ }^{79}$.

A pesar de que estas iniciativas se establecieron hace 25 años, las tasas mundiales de lactancia siguen estando muy por debajo de los objetivos internacionales.

Los 10 pasos para una Lactancia Materna Exitosa: Conocida como Iniciativa Hospital Amigo de la Madre $Y$ Niños. Es una propuesta de UNICEF y la OMS la acoge desde 1993. Propone una metodología e instrumentos para identificar un conjunto de procesos que de cumplirlos, un estacionamiento de salud garantizaría el apoyo a sus usuarias para una lactancia materna exitosa. Versiones revisadas, actualizadas y ampliada. Enero 2006 y $2008^{80}$ ( Tabla 3).

Es muy importante instruir a la madre de una manera práctica las técnicas de amamantamiento, dado que un buen agarre garantiza una lactancia materna exitosa, (Figura 1) así como la lactancia materna a libre demanda y exclusiva.

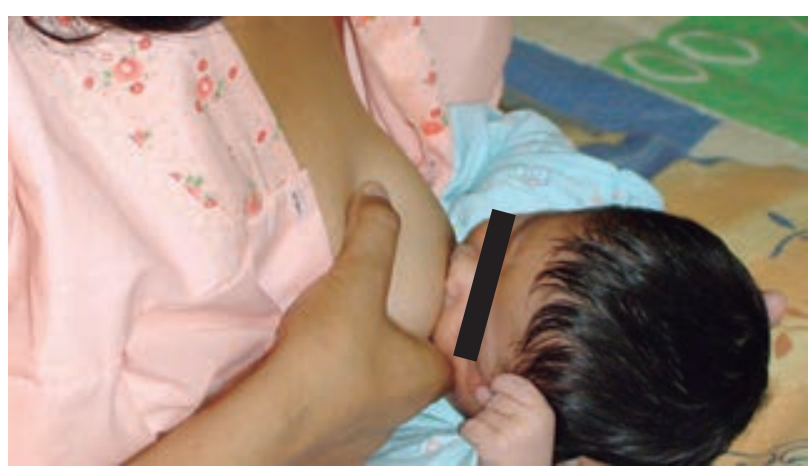

Figura 1. Óptima posición de agarre, asegura una lactancia materna exitosa. 


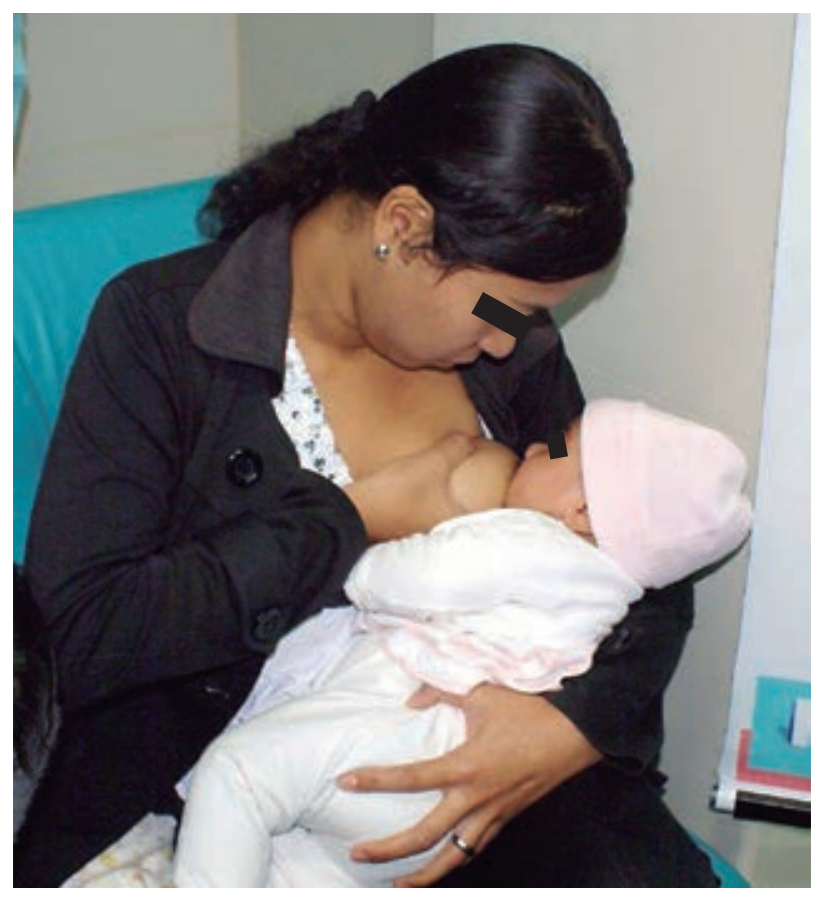

Figura 2. Madre y bebé disfrutando su derecho de amamantar y recibir leche materna.

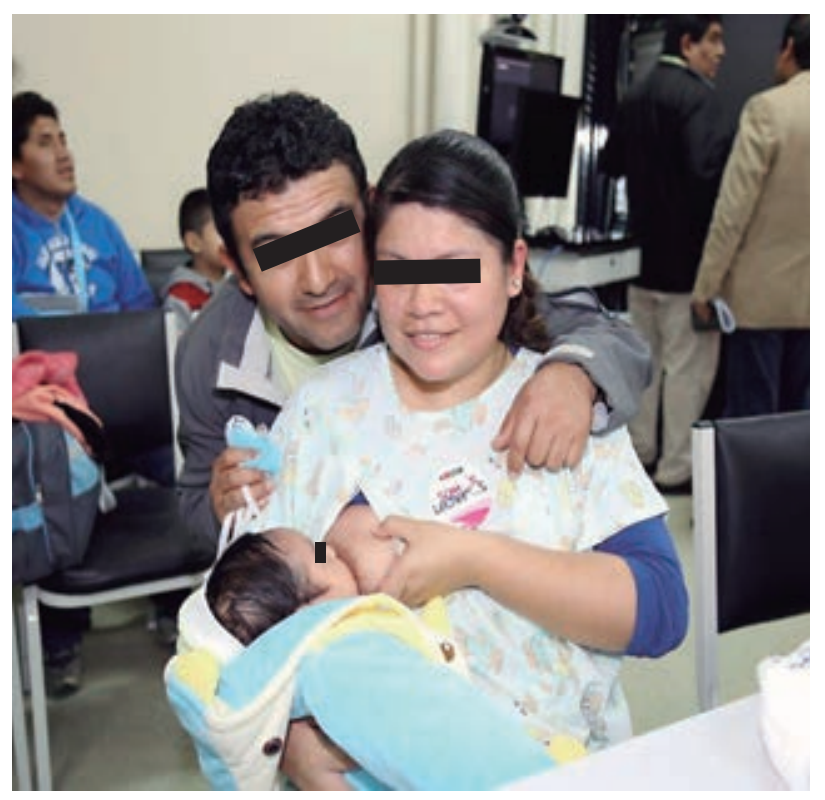

Figura 3: Bebé lactando, familia feliz.

La lactancia debe ser una relación feliz de la madre con su bebé, debe ser disfrutada por ambos (Figura 2), sin dejar de lado al padre que debe de integrarse en esta actividad, acompañando, apoyando, brindando masajes, apoyo moral a la madre, etc. (Figura 3).

\section{CONCLUSIONES}

La leche materna humana no tiene sólo ventajas nutricionales perfectamente adaptadas para el bebé, sino que los resultados de estudios realizados sugieren la existencia de mecanismos que la consideran como la medicina personalizada más específica, en el momento en que la expresión genética está experimentando un ajuste de alta calidad para la vida.

Se recomienda el inicio de la lactancia materna en la primera hora de vida, esta es una oportunidad para producir una impronta en la salud que no se debe desperdiciar, continuar con ella de manera exclusiva durante los primeros 6 meses y luego acompañada de alimentaciòn complementaria.

La lactancia materna es una de las intervenciones de mayor impacto en la salud pública, que proporcionan beneficios para los niños, las mujeres y la sociedad; la promoción, protección y apoyo a la lactancia materna es necesaria para lograr los Objetivos de Desarrollo Sostenible.

El éxito en la lactancia materna no es responsabilidad exclusiva de una mujer; la promoción de la lactancia materna es una responsabilidad social colectiva, en la que el equipo multidisciplinario de salud tiene el rol protagónico.

Financiamiento: autofinanciado.

Conflicto de interés: la autora declara no tener algún conflicto de interés.

\section{REFERENCIAS BIBLIOGRÁFICAS}

1. Nota Editorial. La lactancia materna: alcanzando la necesaria normalidad. Serie 2016 sobre Lactancia Materna. The Lancet. 2016;387:475-504

2. Gartner LM, Morton J, Lawrence RA, et al. American Academy of Pediatrics Section on Breastfeeding. Breastfeeding and the use of human milk. Pediatrics. 2005;115 (2):496-506

3. Jones G, Steketee RW, Black RE, Bhutta ZA, Morris SS. Bellagio Child Survival Study Group. How many child deaths can we prevent this year? Lancet. 2003; 362: 65-71

4. Informe de Centro Nacional de Epidemiología, Prevención y Control de Enfermedades - RENACE PERU 2017, Sem 22.

5. Schanler R, Abrams S. Nutritional composition of human milk for full term infants, Wolters Kluwer. www. Uptodate 2016; $p$ 1-6

6. Gura T. Nature's first functional food. Science 2014; 345 : 747-49.

7. Rassin D, Gaull G, Ráihá N, Heinonen K. Milk protein quantity and quality in low-birth-weight infants. IV. Effects on tyrosine and phenylalanine in plasma and urine. J Pediatr 1977; 90:356.

8. Billeaud C, Guillet J, Sandler B. Gastric emptying in infants with or without gastro-oesophageal reflux according to the type of milk. Eur J Clin Nutr 1990; 44:577.

9. Goldman AS, Chheda S, Keeney SE, et al. Immunologie protection of the premature newborn by human milk. Semin Perinatol 1994; 18:495.

10. Hanson L, Ahlstedt S, Andersson B, et al. Protective factors in milk and the development of the immune system. Pediatrics $1985 ; 75: 172$. 
11. Carver JD, Walker WA. The role of nucleotides in human nutrition. Nutr Biochem 1995; 6:58.

12. Mandel D, Lubetzky R, Dollberg S, et al. Fat and energy contents of expressed human breast milk in prolonged lactation. Pediatrics 2005; 116: e432.

13. Jensen RG. The lipids in human milk. Prog Lipid Res 1996; 35:53.

14. Hemell O, Blijckberg L. Human milk bile salt-stimulated lipase: functional and molecular aspects. J Pediatr 1994; 125:556.

15. Uauy R, Dangour A. Nutrition in brain development and aging: role of essential fatty acids. Nutr Rev.2006; 64: S24-33.

16. Váldez R. Nutrición del recién nacido. I Ed. Ciencias Médicas, Capítulo 12: Lactancia Materna, Cuba 2010, 117-145p

17. Victora C, Bahl R, Barros A, França G, Horton S, Krasevec J and cols. La lactancia materna en el Siglo XXI: epidemiología, mecanismos y efectos a lo largo de la vida. Lancet 2016; 387; 475-90.

18. Azad M, Konya $\mathrm{T}$, Maughan $\mathrm{H}$, et al. Gut microbiota of healthy Canadian infants: profi les by mode of delivery and infant diet at 4 months. CMAJ 2013; 185: 385-94.

19. Yatsunenko T, Rey F, Manary M, et al. Human gut microbiome viewed across age and geography. Nature 2012; 486: 222

20. Gura T. Nature's fi rst functional food. Science 2014; 345 : 747-49

21. Hooper LV, Littman DR, Macpherson AJ. Interactions between the microbiota and the immune system. Science 2012; 336: 1268-73.

22. Latuga MS, Stuebe A, Seed PC. A review of the source and function of microbiota in breast milk. Semin Reprod Med 2014: 32: 68-73.

23. Torregrosa Paredes $P$, Gutzeit $C$, Johansson $S$, et al. Differences in exosome populations in human breast milk in relation to allergic sensitization and lifestyle. Allergy 2014; 69: 463-71.

24. Mayer EA, Knight R, Mazmanian SK, Cryan JF, Tillisch K.Gut microbes and the brain: paradigm shift in neuroscience.J Neurosci2014: 34: 15490-96.

25. Lutter C. ¡El inicio temprano de la lactancia materna: la clave para supervivencia y desarrollo! Boletín informativo OMS 2010: pag 1-8.

26. Eidelman A, Schanler R and cols. Breastfeeding and the Use of Human Milk. Pediatrics 2012;129: e827-e841

27. Sankar MJ, Sinha B, Chowdhury R, et al. Optimal breastfeeding practices and infant and child mortality. A systematic review and meta-analysis. Acta Paediatr 2015; published online Aug 7. DOI:10.1111/apa.13147.

28. Horta B, Victora C. Short-term eff ects of breastfeeding: a systematic review of the benefi ts of breastfeeding on diarhoea and pneumonia mortality. Geneva: World Health Organization,2013.

29. Ip S, Chung M, Raman G, et al; Tufts-New England Medical Center Evidence-based Practice Center. Breastfeeding and maternal and infant health outcomes in developed countries. Evid Rep Technol Assess Rep. 2007;153(153):1-186

30. Ip S, Chung M, Raman G, Trikalinos TA, Lau J. A summary of the Agency for Healthcare Research and Quality's evidence report on breastfeeding in developed countries. Breastfeed Med. 2009;4(suppl 1):S17-S30

31. Chantry CJ, Howard CR, Auinger P. Full breastfeeding duration and associated decrease in respiratory tract infection in US children. Pediatrics. 2006;117(2):425-432

32. Duijts L, Jaddoe V, Hofman A, Moll H. Prolonged and exclusive breastfeeding reduces the risk of infectious diseases in infancy. Pediatrics. 2010;126(1)
33. Sullivan S, Schanler R, Kim J, et al. An exclusively human milk-based diet is associated with a lower rate of necrotizing enterocolitis than a diet of human milk and bovine milk-based products. J Pediatr.2010;156(4):562-567, e1

34. Zutavern A, Brockow I, Schaaf B, et al. LISA Study Group. Timing of solid food introduction in relation to atopic dermatitis and atopic sensitization: results from a prospective birth cohort study. Pediatrics. 2006;117(2):401-411

35. Akobeng A, Ramanan A, Buchan I, Heller R. Effect of breast feeding on risk of coeliac disease: a systematic review and metaanalysis of observational studies. Arch Dis Child. 2006;91(1):39-43

36. Barclay A, Russell R, Wilson M, Gilmour W, Satsangi J, Wilson D. Systematic review: the role of breastfeeding in the development of pediatric inflammatory bowel disease. $J$ Pediatr. 2009;155(3):421-426

37. Rosenbauer J, Herzig P, Giani G. Early infant feeding and risk of type 1 diabetes mellitus-a nationwide population-based case-control study in pre-school children. Diabetes Metab Res Rev. 2008;24(3):211-222

38. Das U. Breastfeeding prevents type 2 diabetes mellitus: but how and why? Am J Clin Nutr. 2007;85(5):1436-1437

39. Rudant J, Orsi L, Menegaux F, et al.Childhood acute leukemia, early common infections, and allergy: The ESCALE Study. Am J Epidemiol. 2010;172(9):1015-1027

40. Kwan ML, Buffler PA, Abrams B, Kiley VA. Breastfeeding and the risk of childhood leukemia: a meta-analysis. Public Health Rep. 2004;119(6):521-535

41. Bowatte G, Tham R, Allen KJ, et al. Breastfeeding and childhood acute otitis media: a systematic review and metaanalysis. Acta Paediatr Suppl 2015; 104: 85-95.

42. Amitay EL, Keinan-Boker L. Breastfeeding and childhood leukemia incidence: a meta-analysis and systematic review. JAMA Pediatr 2015; 169: e151-25.

43. Lodge CJ, Tan DJ, Lau M, et al. Breastfeeding and asthma and allergies: a systematic review and meta-analysis. Acta Paediatr Suppl 2015; 104: 38-53.

44. Vohr BR, Poindexter BB, Dusick AM, et al; NICHD Neonatal Research Network. Beneficial effects of breast milk in the neonatal intensive care unit on the developmental outcome of extremely low birth weight infants at 18 months of age. Pediatrics. 2006;118(1).

45. Vohr BR, Poindexter BB, Dusick AM, et al; National Institute of Child Health and Human Development National Research Network. Persistent beneficial effects of breast milk ingested in the neonatal intensive care unit on outcomes of extremely low birth weight infants at 30 months of age. Pediatrics. 2007;120(4).

46. Furman L, Taylor G, Minich N, Hack M. The effect of maternal milk on neonatal morbidity of very low-birth-weight infants. Arch Pediatr Adolesc Med. 2003;157(1):66-71

47. Hooper LV, Littman DR, Macpherson AJ. Interactions between the microbiota and the immune system. Science 2012; 336: 1268-73.

48. Okamoto T, Shirai M, Kokubo M, et al. Human milk reduces the risk of retinal detachment in extremely low-birthweight infants. Pediatr Int. 2007;49(6):894-897

49. Horta B, de Mola C, Victora C. Breastfeeding and intelligence: systematic review and meta-analysis. Acta Paediatr Suppl 2015; 104: 14-19.

50. Lucas A, Morley R, Cole T. Randomised trial of early diet in preterm babies and later intelligence quotient. BMJ. 1998;317 (7171):1481-1487.

51. Kramer M, Aboud F, Mironova E, et al; Promotion of Breastfeeding Intervention Trial (PROBIT) Study Group. 
Breastfeeding and child cognitive development: new evidence from a large randomized trial. Arch Gen Psychiatry. 2008;65(5):578-584.

52. Lucas A. Long-term programming effects of early nutrition-implications for the preterm infant. $\mathrm{J}$ Perinatol. 2005;25(suppl2):S2-S6

53. Isaacs EB, Fischl BR, Quinn BT, Chong WK, Gadian DG, Lucas A. Impact of breast milk on intelligence quotient, brain size, and white matter development. Pediatr Res. 2010;67(4):357-362

54. Kramer M, Fombonne E, Igumnov S, et al; Promotion of Breastfeeding Intervention Trial (PROBIT) Study Group. Effects of prolonged and exclusive breastfeeding on child behavior and maternal adjustment: evidence from a large, randomized trial. Pediatrics. 2008;121(3).

55. Der G, Batty G, Deary I. Effect of breast feeding on intelligence in children: prospective study, sibling pairs analysis, and meta-analysis. BMJ. 2006;333(7575):945-950

56. Mortenson, E.L. et al. The association between duration of breastfeeding and adult intelligence. JAMA. 2008:287: 2365-2371.

57. Victora C, Horta B, de Mola C, et al. Association between breastfeeding and intelligence, educational attainment, and income at 30 years of age: a prospective birth cohort study from Brazil. Lancet Glob Health 2015; 3: e199-e205.

58. Singhal A, Cole TJ, Lucas A. Early nutrition in preterm infants and later blood pressure: two cohorts after randomised trials. Lancet. 2001;357(9254):413-419m

59. Parikh NI, Hwang SJ, Ingelsson E, et al. Breastfeeding in infancy and adult cardiovascular disease risk factors. Am J Med. 2009;122(7):656-663, e1

60. Owen CG, Martin RM, Whincup PH, Smith GD, Cook DG. Effect of infant feeding on the risk of obesity across the life course: a quantitative review of published evidence. Pediatrics. 2005;115(5):1367-1377

61. Peres KG, Cascaes AM, Nascimento GG, Victora CG. Eff ect of breastfeeding on malocclusions: a systematic review and metaanalysis. Acta Paediatr Suppl 2015; 104: 54 -61

62. Labbok, M.H. Effects of breastfeeding on the mother. PediatrClin North Am. 2001:48: 143-58.

63. Becker S, Rutstein S, Labbok MH. Estimation of births averted due to breast-feeding and increases in levels of contraception needed to substitute for breast-feeding. J Biosoc Sci 2003; 35: 559-74.

64. Krause K, Lovelady C, Peterson B, Chowdhury N, Ostbye T. Effect of breastfeeding on weight retention at 3 and 6 months postpartum: data from the North Carolina WIC Programme. Public Health Nutr. 2010;13(12):2019-2026

65. Bobrow KL, Quigley MA, Green J, Reeves GK, Beral V. Persistent effects of women's parity and breastfeeding patterns on their body mass index: results from the Million Women Study. Int J Obes (Lond) 2013; 37: 712-17.

66. Aune D, Norat T, Romundstad P, Vatten L. Breastfeeding and the maternal risk of type 2 diabetes: a systematic review and doseresponse meta-analysis of cohort studies. Nutr Metab Cardiovasc Dis 2014; 24: 107-15.
67. Stuebe A, Rich-Edwards J, Willett W, Manson J, Michels K. Duration of lactation and incidence of type 2 diabetes. JAMA. 2005;294(20):2601-2610

68. Schwarz E, Brown J, Creasman J, et al. Lactation and maternal risk of type 2 diabetes: a population-based study. Am J Med. 2010;123(9):863.e1-.e6

69. Horta BL, de Mola CL, Victora CG. Long-term consequences of breastfeeding on cholesterol, obesity, systolic blood pressure, and type-2 diabetes: systematic review and metaanalysis. Acta Paediatr Suppl 2015; 104: 30-37.

70. Schwarz E, Ray R, Stuebe A, et al.Duration of lactation and risk factors for maternal cardiovascular disease. Obstet Gynecol. 2009;113(5):974-982

71. Henderson J, Evans S, Straton J, Priest S,Hagan R. Impact of postnatal depressionon breastfeeding duration. Birth. 2003;30 (3):175-180

72. Dias C, Figueiredo B. Breastfeeding and depression: a systematic review of the literature. J Aff ect Disord 2015; 171: 142-54.

73. Chowdhury R, Sinha B, Sankar MJ, et al. Breastfeeding and maternal health outcomes: a systematic review and metaanalysis. Acta Paediatr Suppl 2015; 104: 96-113.

74. Collaborative Group on Hormonal Factors in Breast Cancer. Breast cancer and breastfeeding: collaborative reanalysis of individual data from 47 epidemiological studies in 30 countries, including 50302 women with breast cancer and 96973 women without the disease. Lancet 2002; 360: 187-95.

75. Karlson E, Mandl L, Hankinson S,Grodstein F. Do breastfeeding and other reproductive factors influence future risk of rheumatoid arthritis? Results from the Nurses' Health Study. Arthritis Rheum.2004;50(11):3458-3467

76. Bartick M, Reinhold A. The burden of suboptimal breastfeeding in the United States: a pediatric cost analysis. Pediatrics. 2010; 125(5).

77. Edmond K. et al. Delayed breastfeeding initiation increases risk of neonatal mortality. Pediatrics. 2006:117: 380-386 .

78. Mullany, L.C. et al. Breast-feeding patterns, time to initiation, and mortality risk among newborns in Southern Nepal. J Nutr. 2008: 138: 599-603.

79. León Z, Villanueva C, Dávila C. Banco de Leche Humana: estrategia para la disminución de la morbilidad neonatalInstituto Nacional Materno Perinatal. Rev. Peru Investig Matern Perinat 2016: 5(2) 31-36

80. Chavez de Pop V. La Iniciativa Hospital Amigo de la madre y el niñ@: evidencias científicas de los 10 pasos y avances reciente. Disponible en http://www.Unicef.org/republicadominicana/ DOR_IHAN.pdf. (Publicado Febrero 2013).

\section{Correspondencia:}

Dirección: Jr. Belgrano N 372- Pueblo Libre.Lima-Perú.

Teléfono: 999042084

E-mail: davilacarmen@hotmail.com 